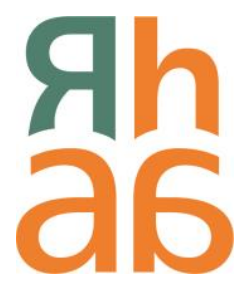

\title{
El crédito en economías de Antiguo Régimen: Algunas cuestiones teóricas y reflexiones sobre la historiografía de la América portuguesa
}

\author{
Credit in Old Regime economies: some theoretical \\ questions and reflections on the historiography of \\ Portuguese America
}

\author{
Tiago Luís Gil \\ Universidade de Brasília \\ Brasília, Brasil \\ tiagoluisgil@gmail.com
}

\begin{abstract}
Resumen
El objetivo de este artículo es considerar algunas cuestiones teóricas relacionadas con el crédito, sobre la base del debate sostenido en la historiografía brasileña en los últimos años. En esa discusión el crédito ha sido bien estudiado, no sólo en obras dedicadas exclusivamente a este tema, sino también en otras publicaciones sobre la economía colonial en general. En este sentido, se propone analizar ese estado de la cuestión en cuatro temas específicos. El primero se refiere a la jerarquía social, extremadamente importante en las sociedades del Antiguo Régimen. El segundo, sobre el llamado microcrédito, con referencia a pequeños prestatarios y prestamistas que manejan pequeñas cuantías. Esta actividad puede revelar elementos fundamentales de las economías de tipo antiguo. También discutiremos aspectos como la confianza y la información, ya que fueron analizados con poca profundidad en la historiografía brasileña sobre el período colonial. Finalmente, proponemos reflexionar sobre el carácter opaco del crédito como objeto
\end{abstract}

Cómo citar este artículo/ How to cite this article: Gil, T. (2021). Crédito en economías del Antiguo Regimen: algunas cuestiones teóricas y reflexiones sobre la historiografía de la América portuguesa. Revista de Historia Americana y Argentina, 56 (1), pp. 103-132. https://doi.org/10.48162/rev.44.003 
de estudio, ya que es un fenómeno muy visible y evidente si - y sólo si consideramos las fuentes disponibles, ignorando los arreglos orales.

Palabras clave: economía del Antiguo Régimen; crédito; confianza; información.

\begin{abstract}
This article addresses some theoretical issues related to credit taking into account the debate conducted in Brazilian historiography in recent years. Credit has been extensively studied recently, not only in works exclusively dedicated to this topic, but in publications about the colonial economy in general. The article highlights the little theoretical debate in Brazilian historiography, stressing four themes. The first concerns the social hierarchy, extremely important in Old Regime societies. The second is on the so-called microcredit, with reference to small borrowers and lenders who handled small amounts. I believe this activity may reveal the capital elements of old type economies. The topics of trust and information will also be discussed, since they were analyzed with little depth in the historiography. Finally, I propose reflecting on the opaque character of credit as an object of study, since it is a very visible and evident phenomenon if only available sources are considered, ignoring oral arrangements.
\end{abstract}

Key words: old Regime Economy; credit; trust; information.

Recibido: 23/03/2021. Aceptado: 23/09/2021

El objetivo de este artículo es discutir algunas cuestiones teóricas relacionadas con el tema del crédito en las sociedades del Antiguo Régimen, teniendo como interlocutores algunos de los trabajos más importantes sobre el tema en la historiografía brasileña de los últimos años. De ese repertorio de contribuciones sobre la economía del Brasil colonial, a pesar del escaso número de investigaciones, destacamos estudios que en las últimas dos décadas han aportado nuevos temas al debate. El crédito fue uno de ellos y no solo hay nuevas obras dedicadas exclusivamente al tema (Santos 2013; Azeredo, 2016; Souza, 2019; Gil, 2020), sino que algunas encaminadas a explicar otros temas, como la producción o la circulación, destacaron la importancia del credito. (Sampaio 2003; Ribeiro 2009; Mathias 2012; Santos 2013; Pesavento 2013; Gil y Pesavento 2014; Pinheiro, 2018; Moraes, 2018). Nuestro punto central se refiere a ese relativamente escaso debate teórico en la historiografía brasileña, y 
puntualizamos cuatro temas que parecen haber sido discutidos superficialmente hasta ahora.

En un importante artículo recientemente publicado, Carrara (2020) hace un balance historiográfico muy amplio sobre los estudios crediticios en el Brasil colonial. Al recuperar aportes desde el siglo XIX y pasar por la historiografía de la primera mitad del siglo $\mathrm{XX}$, el autor hace un recorrido sobre el tópico y presta especial atención a algunos nuevos estudios sobre el crédito realizados en Brasil. Para él, desde principios del siglo XXI, una nueva ola de trabajos ha ido planteando nuevas preguntas y ha utilizado una buena multiplicidad de fuentes seriadas para sus investigaciones. Ante tal diversidad, podríamos encontrar algunos puntos de diálogo entre estos nuevos aportes, especialmente en lo referido a la escasez de divisas, las tipologías de las partes (acreedores y prestatarios), la relación entre crédito y actividad económica y la confianza.

El tema de la escasez de moneda es discutido por Carrara, especialmente a partir de los nuevos estudios de Cerqueira Lima (2012), Puntoni (2017) y Santos (2013) que cuestionan la supuesta falta de efectivo. Gran parte de las quejas ante la escasez constante sería el resultado de la inestabilidad política, la falta de monedas específicas (oro, cobre o plata, por ejemplo, con destino a diferentes mercados) o el resultado de circunstancias cíclicas, escenarios completamente diferentes a la denuncia crónica. Frente a estas novedades historiográficas, fruto de renovadas perspectivas y enfoques innovadores, se pondría en jaque la posición del crédito como "respuesta" a la crisis del efectivo, ya que este último proceso no sería tan común como se imagina.

La tipología de las partes interesadas es otro tema importante considerado por el autor. Además de enumerar las diferentes formas de clasificación de los agentes sociales involucrados en la actividad crediticia, hace especial énfasis en los grandes actores, resultado también de la elección de la historiografía que está observando. Los comerciantes y proprietários de ingenio de azúcar tienen prioridad, especialmente estos últimos, tanto en la historiografía crediticia brasileña como en los documentos de la época, que discutieron animadamente sobre la solvencia de estos personajes.

También analizó el uso potencial del crédito como proxy de la actividad económica. Según el artículo, varios autores señalan una estrecha conexión entre el nivel de actividad económica y la fluctuación del mercado crediticio, 
tanto en su movimiento general como en elementos específicos como la expansión de la moneda. Carrara observa acertadamente que existen muchos otros elementos que podrían interferir en los resultados, tanto en la propia economía como en la forma en que aparece en las fuentes, por lo que se dificulta asociar los dos elementos de manera tan directa.

Finalmente, también consideró el tema de la confianza y Carrara lo aborda junto con el de la escasez de divisas. Gran parte de la historiografía presentaría la "confianza" como el principal elemento de liquidez en la economía portuguesa americana, ya que habría una expectativa de que el material rodante de efectivo fuera limitado. En este punto, Carrara adopta una postura neohobbesiana y minimiza otros factores, especialmente la noción de "palabra", ya que las obligaciones contractuales (visibles en la propia existencia del documento utilizado) serían mucho más expresivas. Estamos de acuerdo con él en que el uso demasiado amplio de la noción de "palabra" es más un obstáculo que una ayuda. Sin embargo, es bien sabido que una parte importante de las operaciones crediticias existían desde hace meses o años en el acuerdo verbal para, en caso de problemas, convertirse en documento. Por lo tanto, los contratos verbales eran importantes y deberían estudiarse a pesar de la dificultad para identificarlos.

Carrara, además, hace una discreta crítica a los autores que dialogan con el pensamiento católico de la época moderna, y destaca que:

Submeter as operações de crédito a uma perspectiva moral obnubila seu verdadeiro conteúdo. A despeito dos preceitos religiosos, havia vozes na mesma época que advertiam quanto à fragilidade do juramento... (Carrara, 2020, p. 37)

Entendemos que el autor no valora las causas de la "obnubilación", pero usaremos la expresión de manera positiva. Muchos factores no económicos, sociales o legales oscurecieron efectivamente el "verdadero contenido" del crédito, e interfirieron en su esencia: el parentesco, la amistad, la reputación e incluso la moral. Aquí es donde radica el interés histórico (e interdisciplinario) de los estudios de crédito. Sin embargo, Carrara tiene razón al señalar este problema. A pesar de la diferencia de opinión, hay una cierta exageración en la forma en que se incorporó la tratadística católica, moderno o incluso medieval, para explicar el comportamiento económico de la era moderna, adoptando muchas veces un perfil idealista, en el cual la tratadística explica el comportamiento social y lo modifica a medida que los filósofos cambian de opinión, sin una circulación de visiones del mundo 
entre los dos extremos 0 incluso reinterpretaciones del discurso "compartido" (Novaes Marques, 2014).

A pesar de esta divergencia, estamos de acuerdo con buena parte de las consideraciones presentadas por Carrara. El punto de discusión no está en el escenario presentado por el autor, sino exactamente está en lo que el texto ignora. Esto sería el resultado de la ausencia de debate historiográfico (en Brasil) sobre ciertos temas que resultan fundamentales para entender el crédito en las economías del Antiguo Régimen ( $\mathrm{y}$, como veremos, incluso en las economías contemporáneas). El primero se refiere a la jerarquía social (extremadamente importante en sociedades como esta); es un tema que se recuperó en la historiografía reciente, pero con algunas lagunas importantes, ya que todavía se pone mucho énfasis en el comportamiento de los grandes propietarios de ingenios de azúcar, por ejemplo, pero no así en los demás participantes de la escala social. Veremos que no se trata de una cuestión de equilibrio o de prioridades.

Otro punto primordial es lo que podríamos llamar microcrédito. Si buena parte de nuestros conceptos están definidos a posteriori y quizás por eso siempre suenan anacrónicos, hacer referencia al microcrédito parece una herejía sin fin. Pero nos referimos a los pequeños acreedores y prestamistas que manejan pequeñas cantidades, especialmente en entornos comunitarios. Esta actividad, si se estudia a fondo, puede revelar elementos fundamentales de las economías de Antiguo Régimen. Hasta cierto punto, esto tiene una conexión directa con el problema de la jerarquía. El tema de la confianza parece haber sido tratado de forma superficial por gran parte de la historiografía, que insistió en la "palabra dada" como un elemento cultural genérico, en un mundo supuestamente controlado por el miedo a lo sobrenatural. La pregunta, está directamente relacionada con otro fenómeno, que apenas aparece en el texto de Carrara y es igualmente discreto en la historiografía: la información (Wasserman, 2018).

Finalmente, reflexionamos sobre el carácter opaco del crédito como objeto de estudio. Es un fenómeno, aparentemente, visible y evidente si - y solo si - consideramos que las fuentes disponibles agotan la historia de su práctica. Gran parte de los estudios de crédito en el escenario actual de la historiografía brasileña consideran el documentado como el crédito en sí mismo e ignoran cualquier intento de buscar prácticas indocumentadas, no solo para negocios orales, sino también para aquellos que solo fueron 
parcialmente documentados y omitieron aspectos cualitativos (cuantificables o no) de importancia.

\section{Jerarquía y crédito en el Antiguo Régimen}

Gran parte de la historiografía crediticia brasileña considera el problema de la siguiente manera: cuando alguien presta dinero, la atención se centra en el acreedor (que tiene dinero para prestar) y no en el prestatario de los recursos. Cuando alguien devuelve el dinero (o no) la atención se centra en el deudor. Y esta cuestión se considera la más superficial, inmediata y vinculada a la estructura de los documentos. Está claro que un acreedor engañado generará documentos para reclamar su pago y un acreedor activo mencionará y destacará a sus deudores. Estamos considerando un uso analítico más directo de la fuente, que es el más frecuente, y necesitamos encontrar formas de superarlo.

Para ello, proponemos invertir el enfoque. Observar al prestatario, en el primer préstamo, y al prestamista, en el momento del pago. El énfasis ya no está en quienes tienen dinero y pueden prestarlo o devolverlo a quienes no lo tienen, en el primer movimiento, o pueden perderlo (o perder aún más) en el segundo. Se podría objetar que, en ambos movimientos, debemos observar a todos los jugadores. Estamos de acuerdo, pero la historiografía ha dado preferencia a algunos como hemos señalado. Invertir el ángulo puede revelar elementos interesantes, aunque sea como recursos analíticos (Sampaio, 2003; Santos, 2003; Mathias, 2012; 2005 y 2013). En sí mismo, el procedimiento de separación de participantes y actos es un dispositivo analítico importante.

Es cierto que los que prestan tienen recursos y deciden a quién prestarán (o no). Pero el hecho de que alguien no tenga los recursos y los obtenga es más revelador. Por otro lado, que alguien devuelva el recurso, aunque no sea natural, es algo relativamente esperado por esa sociedad. Las más interesantes son las acciones sucesivas por parte del prestamista ante el comportamiento del prestatario. Este juego dinámico como herramienta analítica es relativamente raro en la historiografía, lo que da lugar a análisis estáticos y cosificantes. Mathias, por ejemplo, rara vez discute el "mercado crediticio" que estudia y termina cosificando su existencia, como si no fuera el resultado de interacciones sociales, aunque su objetivo fuera exactamente el contrario (Mathias 2012). 
Estos temas son importantes cuando hablamos de jerarquía, ya que uno de los ejemplos más frecuentes de estudios crediticios se refiere al comportamiento de los proprietários de ingenio de azúcar. En cierta medida, esto ocurre por el importante protagonismo que se le dio a estos personajes en la historiografía brasileña, ganando amplios contornos desde Gilberto Freyre. Los proprietários de ingenio de azúcar también parecen actuar en el crédito ya en la documentación descriptiva del período colonial, desde Frei Cardim, pasando por Frei Vicente, Antonil y amplias referencias en el siglo XVIII. Por otro lado, el papel de estos personajes en la economía fue realmente sustancial, no se puede negar. (Ribeiro, 2009; Novaes Marques, 2014; Carrara, 2020)

La importancia de los proprietários de ingenio de azúcar y su protagonismo documental parece haber generado varios problemas. Gran parte de los estudios crediticios se centran en el desempeño de estos actores y ensalzan su endeudamiento crónico y su comportamiento ante este escenario constante. Teresa Marques dedicó un artículo a este tema, con el sugerente título "Eram os senhores de engenho caloteiros?", en el que analiza la estructura mental que permitió ciertos abusos y que se cuestiona en el siglo XVIII (en el plano mental) y en el siglo XIX (en términos de acciones económicas y legislación). Planteamos si no sería una buena solución responder la pregunta si fueran estafadores con un cambio de roles: “¡Pero no por mucho tiempo!”, y destacaría la decisión de los acreedores; o con otra sugerente pregunta: "¿Están mal informados los prestamistas?" (Novaes Marques, 2014).

Marques no es la única que reifica la posición de los proprietários de ingenio de azúcar en el mundo crediticio. Mathias (2012) también lo hace, aunque parece dar protagonismo a los comerciantes que actuaban como acreedores y controlaban las cadenas de deuda. Este autor sostiene que las Minas del siglo XVIII estaban crónicamente endeudadas y los productores estaban en manos de los acreedores. Si eso es correcto, ¿los comerciantes hicieron negocios con los eternos incumplidores? La respuesta puede incluso ser afirmativa, pero este comportamiento debe aclararse mejor, ya que el crédito puede ser escenario de otras tramas sociales. Este tipo de modelo solo es posible en la medida en que se piensa en un sistema estático, donde las posiciones de cada actor son fijas y poco cambiantes. Como bien mostró Sampaio, las fuentes son muy fragmentarias en este sentido y un mismo agente puede actuar como proprietario de ingenio en un 
documento y comerciante en otro, sin mencionar la amplia ausencia de información (Sampaio, 2003).

El peligro de utilizar categorías estáticas es inmenso y ya ha sido señalado por una amplia bibliografía (Cerutti, 1998; Gribaudi y Blum, 1990). Este es el punto central sobre la reificación del papel del proprietario de ingenio. Las personas pueden tener diferentes tasas de aceptación como prestatarios a lo largo de su vida y esto puede tener una relación muy estrecha con la forma en que se presentan. En tiempos de éxito, su credibilidad puede aumentar y también lo hacen los valores que puede asumir. A esto se le llama reputación y es un concepto ampliamente aceptado dentro de la teoría económica, incluso entre los críticos del concepto de confianza (Guinnane, 2005). Es un fenómeno dinámico e inestable (Gil y Pesavento, 2014; Wasserman, 2018).

Sin embargo, existen varios elementos que perturban la percepción de la reputación. Al ser un fenómeno que depende de la construcción colectiva, distintas fuentes de información pueden generar distintas interpretaciones (simultáneas) sobre un mismo tema. Alguien puede saber sobre quiénes son deudores, mientras que otros no. Entre los diversos elementos que oscurecen la percepción de los agentes económicos entre sí, la jerarquía social es uno de los más importantes. Ante un escenario de inestabilidad, la jerarquía social (del Antiguo Régimen) es un elemento de certeza ilusoria y, aunque ilusoria, también práctica, sencilla y barata (Trujillo, 2020) No implica que los agentes actúen de forma irracional, sino que no tienen elementos para discernir en todo momento (la racionalidad limitada es, por tanto, un concepto importante).

Si tales propietarios de ingenio de azúcar inactivos o mineros endeudados lograron mantener su negocio, fue porque su acción individual resultó, al menos, ambivalente. En el límite, podrían ofrecer alguna ventaja no declarada en las fuentes, que solo se notaría en el cruce de muchos tipos diferentes de documentos y, aun así, sería difícil de identificar (Florentino 1997; Fragoso y Florentino 2001). El trabajo de Souza para Río de Janeiro de principios del siglo XIX hace un buen análisis sobre la jerarquía en el Antiguo Régimen y puede presentarse como una buena forma de resolver el problema, aunque en ciertos análisis toma algunas categorías sociales de forma algo estática (Souza 2019). La investigación de Azeredo (2016) también hace una buena reflexión sobre estas categorías. 
En un estudio sobre el mercado caribeño, Jesús Bohórquez destacó el estatus (como capital simbólico y diferente a la reputación) como un elemento que garantizaba cierta estabilidad en la imagen pública de los agentes económicos, frente a la reputación, que, según él, sería más inestable. La reputación podría, en este modelo, perderse, mientras que el estatus sería constante y, sobre todo, transmisible entre miembros de un mismo grupo. Para él, el estatus no debe confundirse con nobleza o hidalguía. Creo que este proceso puede ser muy explicativo para los mercaderes "profesionales" y parece un modelo válido incluso para los forasteros que trabajan en intercambios. Advertimos, sin embargo, que la jerarquía en el sentido de nobleza o hidalguía también aparece en las valoraciones y cálculos de los agentes históricos del Antiguo Régimen y que el modelo presentado por Bohórquez no invalida el concepto de reputación (Bohórquez, 2017).

Creo que es importante estudiar la jerarquía en sus diversas formas, tanto en los términos presentados por Bohórquez como en sus propias manifestaciones del Antiguo Régimen. Todos eran elementos que interferían en la toma de decisiones. Queda por pensar en formas de abordar el problema sin entrar en tautologías, como si el crédito fuera más generoso con las personas de arriba, que estarían en la cima porque tienen más crédito. Es necesario observar el "proceso generativo" - para tomar una expresión del antropólogo Fredrik Barth - del endeudamiento y observar la secuencia de movimientos de cada actor en el contexto del comportamiento de todos los demás agentes económicos tangibles para cada negocio. Para entendernos con los economistas, antes de cuantificar, necesitamos construir un juego (de teoría de juegos) que nos permita saber qué medir. Barth, de hecho, se inspira mucho en los modelos de juego para proponer varias de sus reflexiones metodológicas (Barth, 1967; 1974 y 1981).

El problema de la jerarquía no es simple y no puede evaluarse utilizando un solo tipo de fuente. Carrara, en su artículo, celebra una renovación en los estudios de crédito, basada también en el uso de diferentes fuentes. La celebración es válida, pero aún queda cierta permanencia en el uso de pocas fuentes, salvo por algunas excepciones (Santos, 2013; Azeredo, 2016; Espírito Santo, 2011), especialmente las escrituras públicas (Actas Notariales). Son documentos extraordinarios, sin duda, pero con sus limitaciones y distorsiones. Si se compara con los inventarios post-mortem (para los mismos lugares), parece que las escrituras públicas son muy comunes para las grandes empresas y, en muchos casos, en los que se ha 
establecido algún desacuerdo, obligan a algunas de las partes a exigir el registro documental: promesa de pago incumplida, retrasos, insatisfacción en la mercancía entregada como pago, entre otras posibilidades; Mientras tanto, los inventarios post mortem a menudo incluyen una mayor gama de negocios (Gil, 2020; Carrara, 2020). Las escrituras no incluyen un gran número de operaciones y de tendencias y seleccionan los más vultuosos. Incluso la importante obra de Azeredo (2016) trata los inventarios como meros complementos de las escrituras.

Hay otro problema con las escrituras, al estar llenas de detalles sobre el negocio que se haría, no siempre hablan de las personas que están negociando. No sabemos si se conoce a las partes (salvo que sean familiares), cuál es su actividad económica, entre otra información de elementos que podrían orientar el negocio. Con los inventarios post mortem se dan diferentes escenarios, que suelen contener listas de acreedores y deudores del fallecido. Esta fuente es particularmente interesante, ya que es muy común encontrar referencias personales y calificativas atribuidas a acreedores y deudores, una forma de individualizar a las partes. Lo mejor de todo es que el calificativo adoptado es diacrónico, es decir, válido para esa situación y evita la extrapolación de categorías sociales estáticas.

Al unir varias referencias al mismo personaje en diferentes inventarios, podemos tener una idea de las formas en que se hizo referencia a él, tanto si subió o bajó en términos sociales. Paralelamente, pero con alguna diferencia de tiempo, tendremos un resumen de sus negocios de crédito. Utilizar estos dos datos de forma cruzada puede ser muy útil para evaluar el peso de la jerarquía. Un teniente puede ascender de rango y ser capitán. Esto también es un ascenso personal y puede tener un impacto en su imagen pública. ¿El valor de los préstamos sería proporcional al status conyuntural? Si un capitán pierde su reputación por alguna razón, ¿dejarían de referirse a él como "capitán" y usaría solo su nombre? Las respuestas deben buscarse y no tomarse a priori.

\section{Microcrédito y sus operadores}

Durante décadas, la historiografía brasileña ha prestado atención solo al crédito operado por grandes señores y mercaderes como lo señala Carrara (2020). La historiografía reciente, a pesar de algunas innovaciones, no ha cambiado este escenario, que sigue privilegiando a quienes movieron los mayores negocios (Novaes Marques 2014; Carrara 2020). Sin embargo, 
describir el crédito no es entenderlo. Para eso, es necesario observar las prácticas crediticias en la sociedad en su conjunto, lo que al menos permite ver el lugar de cada uno en el contexto general (Pereira y Borges, 2010). Sería como estudiar la producción ignorando por completo el consumo (pecado común, por cierto).

Sin embargo, existen razones más sólidas para recomendar estudios sobre microcrédito. En primer lugar, el peso del agregado de pequeñas empresas puede ser de gran importancia en el desarrollo regional, como han demostrado muchos estudios recientes (Guinnane, 2001; Ogilvie, 2005; Malaquias, 2014; Valencia, 2018; Gil, 2018). Hay una razón analítica: el desafío de entender las microfinanzas nos obliga a pensar en elementos que parecían estar resueltos para las grandes empresas, pero que de hecho seguían siendo nebulosos. Los costos de transacción pueden ser relativamente altos para préstamos pequeños, lo que nos obliga a preguntarnos: ¿cómo se controlaban a los deudores? Esto sería solo algún ejemplo de las diversas preguntas que deberían hacerse a los grandes prestatarios y que rara vez se plantean en la historiografía brasileña del período colonial. En este sentido, el estudio del microcrédito podría plantear buenos interrogantes al gran crédito.

Se destacaron los estudios de microcrédito a partir de estudios de experiencias concretas como el del Grameen Bank, en Bangladesh, en la década de 1970. A lo largo de la década de 1990, el interés por estas iniciativas creció y motivó la política económica de varios países y regiones del mundo. La idea del Grameen Bank era proporcionar préstamos de bajo valor con servicios de bajo costo a las comunidades pobres, con la expectativa inicial de simplemente eliminar la actividad de los prestamistas oportunistas. Sus objetivos se han vuelto más complejos hasta el punto de convertirse en un corpus de referencias sobre microfinanzas y sobre el impacto en el desarrollo local (Yunus, 2004).

En un ensayo de 1990, Joseph Stiglitz trató de sintetizar el elemento exitoso del Grameen Bank, y llamó la atención sobre el bajo costo del control utilizado por el sistema. La clave del éxito, para Stiglitz, sería el monitoreo entre pares (peer monitoring), mediante el cual las personas de la comunidad monitoreaban los gastos de los prestatarios y la devolución de recursos. La estrategia fue elegante: siempre se realizaban préstamos a grupos, en los que cada participante indicaba a un vecino como garante. Los integrantes del grupo solo pudieron tener un nuevo préstamo cuando 
todos los demás hubieran pagado las cantidades originalmente asignadas. Las relaciones comunitarias garantizarían el control y las sanciones por comportamientos oportunistas y seleccionarían con anticipación a los participantes por su reputación, con base en la amplia información que tienen disponible por vivir en proximidad ( $\mathrm{y}$ en cierta medida, en comunidad). El riesgo moral se compartiría con la comunidad, sin que esto condujera a un aumento de los servicios (Stiglitz, 1990).

Como podemos ver, el tema central es la observación del proceso en su conjunto: préstamo, control, pago, nuevo préstamo y las relaciones entre las partes. Aquí cobra fuerza la idea de pensar con la ayuda de la teoría de juegos o el concepto de proceso generativo. Lo interesante, sin embargo, está en el diseño del sistema, que se basa en prácticas que ya existen en la comunidad y depende de ellas. El Grameen Bank no creó el control comunitario del que necesita, aunque en gran medida ha maximizado ese control al diseñar su plan. Las relaciones personales y la forma en que circulaba la información eran elementos ya disponibles en el contexto y que fueron utilizados por Yunus; esto, por supuesto, en la experiencia acumulada que tuvo el experimento a lo largo del tiempo.

Los resultados del Grameen Bank fueron muy discutidos y puestos a prueba, especialmente con respecto a la viabilidad del experimento fuera del contexto propuesto originalmente. Incluso el ensayo teórico de Stiglitz fue cuestionado, ya que su modelo no había sido probado adecuadamente con evidencia empírica. Una de estas preguntas provino del historiador económico Timothy Guinnane, quien buscó en la documentación privada de las cooperativas alemanas a fines del siglo XIX y principios del XX una forma de encontrar respuestas a la pregunta sobre el efecto potencial del control mutuo. Su investigación, entonces afirmaba, "supports the view, widespread today, that microcredit institutions that are carefully designed to take advantage of local community ties can provide loans on terms that are impossible for conventional banks" (Guinnane, 2001, p. 387).

El tema que surge del microcrédito es que estos estudios basan su análisis en observar los efectos de los lazos comunitarios. Guinnane incluso hace estimaciones de distancia geográfica y concentración urbana de algunos asentamientos para evaluar el peso de las interacciones en las concesiones y negociaciones crediticias, si el plazo aumentó, si el interés fue mayor, si se hicieron denegaciones de préstamos, entre otras posibilidades, encontrando gran evidencia sobre este efecto. Una imagen similar 
compartió Stiglitz cuando habló de las relaciones vecinales y tuvo en cuenta por supuesto, las enormes diferencias en el contexto de cada caso.

Sin embargo, no tomamos este elemento como una certeza evidente sino como un problema de investigación a considerar. Así, planteamos dos preguntas: ¿cómo fueron las relaciones comunitarias en la América portuguesa y en qué medida podrían lubrificar el negocio crediticio? y ¿de qué manera estas relaciones comunitarias afectaron a grandes prestamistas y grandes prestatarios, sabiendo que la noción de redes de mercado (desde Braudel) y la mencionada "cadena de endeudamiento" está muy consolidada en la historiografía? En esta última pregunta, ¿no sería más apropiado ubicar a cada proprietario de ingenio dentro de su contexto relacional en lugar de generalizar el efecto del calificador?

Sabemos muy poco sobre los entornos relacionales de la América portuguesa y parece arriesgado reproducir la idea, visible en Guinnane y Stiglitz, que da por sentado que la información sobre el comportamiento económico es abundante en las comunidades. Sería importante observar y comprender el tejido de las relaciones personales y su dinámica en pueblos y ciudades, pero también en las principales rutas comerciales. No se trata de estudiar las redes de determinadas familias, algo que puede ser útil, pero no suficiente. Necesitamos tener un sentido general de los flujos de interacción y saber, por ejemplo, si la élite de una determinada aldea interactúa más con sus parientes e iguales de otros lugares o con la población "común" de donde vive. Si los detentores de valores consignables desconocen el interés y comportamiento de sus vecinos potencialmente interesados en préstamos, ¿qué condiciones habría para que el mercado funcione? Por eso, los registros parroquiales, por ejemplo, pueden ser valiosos. El trabajo de Carlos Malaquias (2014) obtuvo respuestas muy útiles en esta direccíon. Al igual que el estudio de mujeres en el crédito, realizado por Azeredo (2016) e indirectamente por Moraes (2018).

Asimismo, es necesario conocer las alternativas relacionales disponibles para los grandes prestatarios. Podían acceder a varios prestamistas pequeños y medianos u obtener una buena cantidad de alguien que tuviera la integridad de los recursos pero, para eso, sería necesario tener información y relaciones. Estos dos factores tienen características que dificultan el estudio: son dinámicos y multifacéticos. Cuando Guinnane habla de cómo la comunidad tiene información barata sobre sus miembros simplifica todos los conflictos internos y reordenamientos que pueden ocurrir 
en las localidades. Esto se debe a que la comunidad genera conflictos con la misma intensidad que genera vínculos (Bacellar, 1997; Levi, 2000; Imízcoz Beunza, 2004; Valencia, 2018).

Antes de pasar al siguiente punto, es pertinente enfocarnos en los pequeños prestatarios y prestamistas. Es comprensible la escasa atención puesta en estos grupos, ya que son discretos en la documentación. Sabemos poco de ellos y realmente sería muy difícil seguir alguna serie de préstamos y pagos realizados 0 incluso cuentas pendientes en establecimientos comerciales, muy común en los inventarios post mortem. Más raro aún sería encontrar información sobre el motivo del préstamo, ya sea para alguna actividad económica o para consumo. La única alternativa, entonces, pasa a ser el intensivo cruce de fuentes, en el que el uso de registros parroquiales puede ser una solución mínimamente satisfactoria. ¿El parentesco espiritual tiene alguna relación con los negocios? ¿Preferiría la gente hacer negocios con sus compinches? ¿Elegirían compadres acordes con sus proyectos económicos? Creo que las respuestas a estas preguntas deben darse en términos coyunturales y en contextos muy específicos, sin desconocer los elementos macroeconómicos generales (Malaquias, 2014).

Al volver rápidamente al tema de la jerarquía, no podemos considerar a los operadores de microcrédito como una masa homogénea. Es necesario buscar, en la diversidad de sus operaciones, diferentes niveles de posibilidades. Para empezar, no todos los pobres tenían el mismo crédito y ciertamente tenía sus matices. ¿Tienen los hombres la misma capacidad de pedir prestado que las mujeres? ¿Oficiales mecánicos como los que no tienen trabajo? ¿Los esclavizados por un señor poderoso tendrían el mismo perfil que los del señor de un solo cautivo? ¿Dónde se endeudaron? ¿En la bodega a la que iban todos los días? ¿Estarían en condiciones (y con buena reputación) para comprar un préstamo en otra parte de la ciudad? Si es así, ¿hasta dónde podría llegar? Y con esto pasamos al tema final de nuestro argumento: la relación entre información y confianza.

\section{Confianza, información y control}

Antes de profundizar en el problema, quisiera aclarar un punto quizá menos importante pero que hay que abordar. Carrara busca minimizar el significado de confianza y, entre sus argumentos, comenta que: 
(...) não constitui qualquer excentricidade da sociedade colonial os potenciais credores fundarem suas decisões sobre a concessão de crédito em informações sobre relações de amizade, parentesco ou nas distinções sociais dos eventuais mutuários. Tais informações continuam nos dias de hoje a exercer influência sobre as operações de crédito; cabe sempre, contudo, ao potencial credor, confiar ou não nelas para conceder ou não o crédito (Carrara 2020, p. 37).

Por un lado, no creemos que nadie haya dicho que fuese específico y por otro lado, si utilizamos este criterio, los hombres de la antigüedad también producían, comerciaban y consumían. Hay muchas formas de establecer relaciones de amistad, parentesco y distinciones sociales. Comprender cómo este crédito afectó en cada época es dar contexto a prácticas muy antiguas, entre las que podemos incluir la familia, la educación y muchas otras formas más ancestrales, pero que se van rehaciendo en cada momento. Curiosamente, esta es la única parte del artículo de Carrara en la que se mencionan estos temas, que vienen a ser refutados de inmediato.

El tema central de Carrara con respecto a la confianza es negar el peso de la "palabra dada" y minimizar el rol de la confianza en el crédito, dando la debida importancia a las garantías e instrumentos legales que permitan el cobro de deudas, es decir, el prestatario se elige por su capacidad de pago y por la capacidad de cobranza del acreedor. El autor se pregunta: "si la palabra dada era tan valiosa para el crédito, ¿por qué entonces recurrir a registros escritos de transacciones?" Si la comparación con el presente sigue siendo válida, muchos préstamos de hoy, incluso los grandes, no dejan documentación escrita, salvo algún recibo bancario dudoso. En ese momento no fue diferente y no se registraron todas las transacciones (incluso las expresivas). Esto es fundamental para entender esa sociedad, ya que diferentes grupos a lo largo del tiempo optan por silenciar diferentes fenómenos cotidianos.

Las reservas de Carrara son comprensibles y compartibles hasta cierto punto. No es posible discutir sobre el poder de la "palabra" como si fuera una panacea. Esto a menudo significa creer en las formas superficiales que la gente ha creado en el pasado con otros fines, incluso mediante estrategias. Bohorquez (2017) sostiene que en la documentación de los comerciantes que trabajaban en el Caribe a fines del siglo XVIII, la "confianza" aparece como una herramienta retórica y el elemento que motivó la toma de decisiones fue el estatus de las partes, al que ya hemos 
aludido. Muchas veces la documentación habla de confianza y simplemente no podemos creer que fuera así.

Existe un largo debate teórico sobre este tema que puede ser importante para completar los enfoques ahora en curso en la historiografía brasileña. Las condiciones para el surgimiento del concepto de confianza se remontan al artículo seminal de Akerlof, en 1970, en el que discutió la noción de asimetría de información y tomó el mercado de autos usados como punto de partida. En ese artículo, Akerlof concluyó que lo que estaba en juego eran "economic models in which 'trust' is important. Informal unwritten guarantees are preconditions for trade and production" (Akerlof, 1970, p. 249). La idea era que, en el mercado, las partes tuvieran diferentes niveles de información y que esto pudiera favorecer a los oportunistas o, en última instancia, hacer inviable el mercado. Un vendedor de autos usados sabe si el vehículo está en buenas condiciones o no, mientras que el comprador no está seguro.

El debate sobre la confianza continuó en muchas direcciones y surgió en muchas otras, ya que no todos los enfoques parten del artículo de Akerlof. Kenneth Arrow (1974) también dejó espacio para el tema de la confianza en "Los límites de la organización". Otro libro importante apareció en 1988, editado por Diego Gambetta, quien abrió la discusión de manera más efusiva. Gambetta y los demás autores que participaron en la colección no estaban completamente seguros de la noción "elusive" de confianza y buscaron evaluar en detalle su significado e impacto. Las respuestas al problema fueron variadas y a pesar de la duda, algunos autores incluso consideraron la confianza como un "lubricante" (retomando Arrow) que solucionaría los problemas de fricciones generadas por la asimetría de información (Dasgupta, 1988, p. 64).

En un artículo algo crítico sobre la noción de confianza, Williamson (1993) demuestra hasta qué punto este concepto es, en la mayoría de los casos, reemplazable por otros (mejor definidos) de riesgo y costo de transacción, basados en la idea de racionalidad limitada y oportunismo. El autor propone repensar el uso del término "confianza" para el comercio y dejarlo más apropiado para lo que puede ser útil: la confianza personal e institucional. La confianza personal estaría ligada a los intereses inmediatos del sujeto, la familia, los amigos y los amores y, a diferencia de la asociada a los conceptos de riesgo, se podría realizar de forma no calculada, sin seguimiento y en base a predilecciones. La confianza institucional sería apropiada para ciertas prácticas sociales, el término institución se piensa en 
términos de North (1994) y Zucker (1977). En este marco, habría instituciones como redes de relaciones, cultura política, regulación, cultura empresarial y profesional y elementos culturales más amplios, como en el caso de que los habitantes de un país sean más confiables que los de otro.

Incluso con la forma rigurosa y algo escéptica en que Williamson aborda el tema, hay espacio para pensar teóricamente sobre prácticas sociales muy diferentes. El autor no resume las prácticas económicas (desde el presente) hasta el contrato, el seguimiento y la posibilidad de establecer sanciones. Existen varias otras formas de interacción económica que se pueden abordar desde el punto de vista de la propia confianza, aunque para él, esto no sirve para pensar en el mercado, para lo cual existen herramientas analíticas más adecuadas, como el costo de transacción y el riesgo. Sería interesante pensar en qué medida estos diferentes ámbitos pueden coincidir $y$, en cierta medida, provocar escenarios difíciles de explicar. Sin embargo, según Williamson, incluso cuando un agente del mercado actúa de manera apasionada, esto puede tenerse en cuenta al calcular el costo de transacción. ¿Serían estas cuestiones tan separables en una sociedad del Antiguo Régimen?

Otro crítico del concepto de confianza es Timothy Guinnane (2005), quien publicó un interesante artículo titulado "Trust: a concept too many", en el que argumentó que a pesar de algunos avances interesantes realizados por investigadores en historia y ciencias sociales con el uso del concepto de confianza, en la gran mayoría de los casos su empleo fue vago y terminó creando más confusión que claridad. Para él, la confianza sería un concepto superfluo y debería ser reemplazado por el uso conjunto de las nociones de información y control (control, en el original: "ability to impose sanctions"). Otros factores serían menos importantes o podrían incluirse bajo ese paraguas. Lo importante para el prestamista es si el prestatario pagará. Tampoco se trata de poder pagar, sino de si tiene los medios para garantizar el pago, si el deudor tiene recursos o no.

Guinnane, sin embargo, no ignora otros elementos, sino que los observa desde el punto de vista de esos dos conceptos principales. Las redes sociales y la familia, por ejemplo, se pueden descomponer en información y control. La información es abundante en la familia, así como (disculpe la repetición) información sobre cuáles serán las sanciones en caso de comportamiento indebido. Cuando se trata de información, no solo debe pensar en quién tiene recursos y quién los necesita, solo para recordar el 
estudio de Rosenthal, Hoffman y Postel-Vinay. (2000), sino también sobre el comportamiento de las personas, las formas en que castigan a quienes se comportan de manera inapropiada o sorprenden a sus socios comerciales. En este sentido, la idea de "información" cobra una fuerza inmensa, algo que estaba implícito, pero no tan valorado en otros textos, salvo en el célebre artículo de Akerlof.

La importancia de la información podría, según Guinnane, adquirir aires aún mayores con la proliferación de noticias sobre el mal comportamiento de un determinado individuo como forma de sanción, es decir, los dos conceptos, aunque separables, podrían formar parte de un mismo proceso. Aquí nuevamente entran elementos de las redes de relación, no como un elemento explicativo, sino como un entorno para la proliferación de información, control y sanciones, lo que Pesavento llamó interdependencia (Pesavento [sd]). Elizabeth de Souza busca construir este entorno, tomando no solo las fuentes inmediatas de crédito, sino los anuncios de los diarios que trataban de las relaciones económicas (aviso de muerte y citación de acreedores, de quiebra, etc.). Este tipo de análisis parece fundamental para entender el contexto social del crédito en una economía (Souza 2019). También es interesante el uso que Pereira y Borges (2010) hicieron a partir de los registros de audiencia de jueces ordinarios, y observaron el mercado desde un punto de vista completamente diferente.

El mismo marco puede evaluarse para las comunidades, por ejemplo, como vimos en un estudio anterior de Guinnane. Lo que no queda claro en este autor (como, de hecho, en muchos otros) es sobre el efecto negativo -de producir ruido e información falsa o exagerada- que puede generar una red, una comunidad o una ciudad. De cualquier manera, comprender cómo funciona la información es un elemento esencial para comprender el crédito. Un caso muy interesante sobre los límites y problemas provocados por la propia comunidad es el estudio de Francesca Trivellato (2009) en el que destaca redes de comerciantes que mantuvieron interacciones comerciales en Livorno en la era moderna. Al analizar la densa red de comerciantes judíos bajo la protección de los Medici, Trivellato observa la construcción de fuertes lazos de confianza con "extraños", quienes luego demostraron ser buenos socios y se comportaron de manera más predecible y cooperativa que los internos del grupo étnico, lo que debilita el punto central de Guinnane. Sin embargo, incluso aquí el tema de la información fue fundamental y la autora reconstruyó en detalle no solo los flujos de circulación de la noticias, sino que además realizó un estudio denso de 
posibles formas de correspondencia entre comerciantes, con el fin de comprender lo dicho, con qué intensidad, cómo y para quién (Trivellato 2009).

El tema de la confianza puede haber sido tratado superficialmente y sin mucha atención al debate que acabamos de ver en la historiografía brasileña, pero el problema de la circulación de la información es completamente ignorado por la misma historiografía, con muy pocas excepciones (Pesavento, 2013). No significa que la idea no está en los autores que se ocupan del crédito, sino que aparece de una forma determinada, sin discutir su significado, se ignoran diferentes matices e intensidades de flujo $y$, principalmente, diferentes rutas, escalas y velocidades de circulación de la información (Mathias 2012; Santos 2013). Se puede objetar que no existen elementos en las fuentes que usualmente se ocupen de créditos para estimar estos datos, de modo que debemos pensar en posibles formas de entender estos fenómenos, a través de fuentes y métodos que permitan estimar mínimamente los límites de los desplazamientos sociales y sus velocidades comunes, y teniendo en cuenta la jerarquía social, que ciertamente debe tener un peso en el acceso a las noticias. El cruce obsesivo de las más diversas fuentes puede ser una solución, aunque laboriosa. No podemos evaluar el peso del crédito utilizando las imágenes deformadas producidas con fuentes aisladas. Se necesita un caleidoscopio.

\section{Opacidad crediticia}

El crédito stricto sensu puede ser un fenómeno aparentemente fácil de observar y cuantificar, a través de ciertos indicadores y documentos. Los elementos que guían la toma de decisiones, sin embargo, no son tan transparentes. No en vano, dos importantes investigadores ya mencionados, Stiglitz y Akerlof, son economistas teóricos. Cuando Akerlof alude al mercado de autos usados, no hizo hojas de cálculo con anuncios de periódicos ni asistió a ferias de autos: usó la abstracción para pensar en problemas difíciles de responder. Por su parte, Stiglitz no fue a Bangladesh a analizar las cuentas del Grameen Bank. Ambos partieron de la observación general de puntos ruidosos en la teoría a partir de insights. No haremos una defensa intransigente de esta práctica, que también tiene sus límites y problemas, pero los historiadores a menudo carecen de este ejercicio. Con esto no restamos importancia a las fuentes, sino propiciamos un uso más crítico del material empírico. Además, parece apropriado 
examinar sobre hasta qué punto las reflexiones teóricas de otras disciplinas pueden ser oportunas para el debate sobre el crédito en la historiografía.

La toma de decisiones es un tema fundamental para comprender las entrañas del crédito. En un estudio dirigido a descifrar este comportamiento, Joyce Berg, Dickhaut y McCabe (1995) organizaron un experimento que aisló a dos grupos de personas en diferentes habitaciones y permitió a los individuos ofrecer dinero de una habitación a otra, con la posibilidad de retribuir, pero sin esta obligación, y los valores eran parte del juego siendo proporcionados por los investigadores. Cuando los valores salieron de una habitación y llegaron a la otra, el equipo de Berg los triplicó y todos los participantes lo sabían. La idea era observar si, ante esta "ganancia", los destinatarios devolverían algo o se llevarían todo. Los resultados obtenidos apuntaban a un fuerte peso de la idea de reciprocidad y de la noción de reputación, ya que una parte expresiva de las personas devolvía sus ganancias.

Con base en el mencionado juego, Glaeser, Laibson, Scheinkman, Soutter (1999) llevaron a cabo una versión más profunda del experimento y expandieron significativamente el número de personas involucradas, para lo cual utilizaron también grupos con diferente etnia, edad, género y otras variables. El resultado reforzó la hipótesis de Berg, Dickhaut y McCabe, al destacar que no existen diferencias muy significativas entre los diferentes grupos, salvo que los hijos únicos eran menos favorables a la devolución del dinero. Otras tantas investigaciones se realizaron teniendo en cuenta la propuesta original de Berg, como la contribución de Johnson y Mislin (2011).

Este tipo de experimento controlado de "laboratorio", con reglas claras y sin otro tipo de interacción que los intercambios propuestos, puede y debe tener limitaciones. Los autores buscaron resolver los puntos anteriores introduciendo una dosis de "pasado" (en busca de reputación) representados por diferentes colectivos y creyeron que la serie de transferencias de dinero y remuneración crearía condiciones similares a las de la vida exterior. No es necesario insistir en lo insuficientes que son estos elementos en comparación con los casos cotidianos. Estos experimentos buscan, sin embargo, obtener información sobre la toma de decisiones que son muy difíciles de conseguir. Los documentos escritos dejan muchos vacíos y pueden resaltar elementos que ni siquiera fueron considerados, por las más diversas razones, como vimos en el estudio de Bohórquez (2017). 
Muy a menudo, los sociólogos tienden a adoptar otra estrategia para obtener las mismas respuestas, con la ayuda de formularios entregados a los operadores económicos (Korczynski, 2000; Burt, 2001). Este método ya ha sido ampliamente criticado por varios economistas que señalan que no necesariamente existe una conexión entre lo que el agente económico hace, piensa que hace y dice que hace (Williamson, 1993; Manski, 2000). Sería realmente difícil seguir -hombro con hombro- a los tomadores de decisiones en el momento en que están decidiendo, en un antropología del comportamiento económico, porque ese no sería un retrato más privilegiado del grupo. La abstracción sigue siendo una de las mejores herramientas, en el sentido de pensar más allá de lo que es inmediatamente visible.

La reflexión que exalta la distancia entre lo que se piensa, se habla y se hace podría servir para considerar las fuentes históricas que utilizamos. Las promesas de confianza deben ser tomadas con cuidado, como bien nos recuerda Carrara. Pero eso no es suficiente, ya que el contexto de la toma de decisiones debe considerarse más detenidamente. Esto requiere seguir de cerca a los tomadores de decisiones y no cuantificarlos como si estuvieran todos en la misma posición. Esto requiere un cambio de perspectiva más centrado en la microeconomía y con un enfoque interdisciplinario, que ponga en diálogo la historia, la economía, la antropología, la sociología y la psicología.

Gran parte de los estudios de crédito en la historiografía brasileña sobre el período colonial se centran en tres tipos de fuentes, en el siguiente orden de uso: escrituras públicas, juramentos del alma e inventarios post-mortem. Todas son fuentes muy adecuadas para este tipo de estudios y, por supuesto, todas tienen sus límites, como bien saben los autores y han destacado en sus estudios. Aunque esto es visible en gran parte de las investigaciones sobre el tema, la reflexión sobre las fuentes y sus límites sigue siendo discreta (Sampaio, 2003; Santos, 2003, 2005 y 2013; Ribeiro, 2009; Mathias, 2012).

Cuando el crédito funciona perfectamente, no puede dejar rastro de su existencia. Un movimiento de préstamo entre amigos, tanto ayer como hoy, no suele dejar pruebas. Y cuando se van, no nos permite conocer una parte expresiva de su significado. Cuando vemos que alguien transfiere recursos a otra persona, imaginamos que el prestamista tenía estos recursos disponibles y no necesitaba, por ejemplo, compartir una parte con un amigo cercano. Sabemos muy poco sobre este submundo de préstamos no 
registrados que realmente existió. $Y$ parece imprudente excluirlos 0 minimizar su peso basándonos en nuestro desconocimiento.

Quizás una forma de superar estos problemas es adoptar prácticas de investigación contrainductivas, como sugiere Feyerabend (2011), que se puede llamar pluralismo metodológico. Esto implica considerar planteamientos hipotéticos, experimentos, procedimientos contrafácticos y juegos como herramientas útiles para la reflexión, así como la lectura de textos y la cuantificación, esta última, en varios niveles. En este sentido, una práctica interesante puede ser utilizar las fuentes como evidencia de sus propias limitaciones. Solo para dar un ejemplo mínimo y muy básico, los historiadores del crédito (Sampaio 2003; Ribeiro 2009; Mathias 2012; Santos 2013; Pesavento 2013; Gil y Pesavento 2014; Azeredo 2016; Souza, 2019; Gil 2020) con frecuencia ponen demasiado énfasis en lo que dice la fuente y terminan ocultando todo lo que silencia, algo que se puede observar comparando y contrastando los documentos entre sí.

Las escrituras son un buen estudio de caso para algunas reflexiones sobre esto. Varias encuestas crediticias en la América portuguesa utilizaron estos documentos (Ribeiro 2009; Santos 2005; Pesavento 2013; Mathias 2012). Son fuentes interesantes, ya que dan los nombres de las partes que realizan un "crédito", detallan las condiciones: plazo, intereses, garantías y otro tipo de información, como la finalidad del préstamo, por ejemplo. Si se analizan detenidamente, revelan un elemento que pone en duda su alcance: no siempre son el resultado de un negocio iniciado en ese momento o una promesa de concesión basada en un registro escrito. La mayoría de las veces, son documentos que registran transacciones anteriores que no tuvieron un desarrollo esperado por alguna de las partes. Algunas transacciones solo aparecen en forma de escritura pública después de semanas, meses o incluso años.

Por otro lado, cuando comparamos los valores medios de las escrituras públicas con otra fuente que habla de créditos, los inventarios post mortem, por ejemplo, tenemos claro que el promedio de las transacciones descritas en las escrituras es mucho mayor que el de los inventarios. Esto se debe a que las escrituras se realizan en condiciones muy especiales: negocios que han envejecido mal, impagos o montos excesivamente altos en comparación con todos los demás negocios que no estaban registrados en escrituras, sino en documentos privados firmados por las partes o, tal vez, solo con base sobre el pacto oral. Por lo tanto, hay una gran parte de 
negocios de los que nunca tendremos ni idea. No quiero decir que necesitemos conocerlos a todos, pero quizá esta tajada desconocida no tendría, por ejemplo, un impacto en la acumulación de recursos que permitirían otros negocios más grandes; o que se trataba de una demostración de confianza más evidente, por ejemplo. Por último, una parte importante de la historia, la del microcrédito, está oculta a nuestra vista y sin explicación. Como intentamos demostrar, esta parte del negocio es relevante no solo por su existencia, sino por la forma en que puede hacernos revisar nuestros modelos explicativos.

Una vez que se hace esta advertencia sobre la opacidad del crédito, creo que existe un aparato conceptual importante en antropología que puede ser útil para resolver estos problemas. Este es el concepto de "esferas económicas", creado por Paul Bohanann (1959). Sugiere que no todos los bienes y servicios se comercializan en los mismos mercados. En algunas economías, por ejemplo, la mano de obra puede ser objeto de un mercado, mientras que en otras no entra en este ámbito y el trabajo está regulado por otras reglas (servidumbre, voluntariado, etc.) La caridad tampoco sería parte de la esfera del mercado, ya que sus costos no están dados por la oferta y la demanda, solo por dar algunos ejemplos. Creo que el crédito también tiene diferentes esferas y debe pensarse de esta manera.

Ciertamente existe un ámbito de los negocios locales, internos a las comunidades, donde el hecho de que las personas se conozcan es muy relevante. Hay otro ámbito, gestionado por profesionales, el de los intercambios regionales, donde pocos tienen suficiente información para actuar. Y habría, al menos, un tercer ámbito donde trabajarían profesionales de alto calibre, especializados o no y con acceso a información que no sería para todos y costaría caro. Son preguntas que no están en las fuentes, mas bien nos obligan a hacer abstracciones útiles para pensar de forma contrainductiva, haciéndonos ver crédito incluso cuando este suele ser más discreto. En este sentido, el análisis de redes sociales puede constituir una metodología útil para esta problemática, no simplemente para saber quién era amigo de quién, sino para mapear la morfología de los préstamos y conocer la intensidad de sus flujos. Sería como tomar el conjunto de negocios de forma integrada, pero no a través de promedios, sino a través de un esquema complejo (Gil, 2015; Wasserman, 2018). 


\section{Conclusión}

El artículo buscaba señalar algunos problemas generales observables en la historiografía crediticia brasileña de la época colonial, enumerados en cuatro puntos: la jerarquía como elemento "perturbador" del crédito, la ausencia de énfasis en las pequeñas empresas (microcrédito), la falta de interés en el debate teórico sobre la confianza y la información y el poco relieve que se le da al crédito indocumentado. Todos estos elementos se cruzan y demuestran un relativo desinterés por el debate teórico que surge de la economía y la antropología económica. Relativo, pues hay varios ejemplos de citas de autores en estos campos del conocimiento, pero pocas veces se confrontan o dialogan. Existe una gran predilección por los debates sobre el peso del pensamiento (medieval y moderno) sobre las acciones de los agentes sociales en la economía de Lusa América. Si no se quiere evitar este debate, tampoco parece válido utilizarlo como clave explicativa principal. Creo que los elementos que hemos enumerado han resultado ser interesantes como problemas de investigación y parte de ellos cuestionan el peso de ese pensamiento en las operaciones crediticias en las economías del Antiguo Régimen.

Finalmente, existe una aceptación generalizada (demasiado, diría yo) de los historiadores del crédito sobre la prevalencia de los contratos escritos en esa economía, demostrada por el uso de estas fuentes sin mucho debate y sobre su valor como prueba material de la necesidad de garantías. Esperamos haber demostrado que muchas operaciones - y lamentablemente no sabemos cuántas - no pasaron por estos arreglos documentados, lo que nos sugiere la existencia de una gran esfera silenciosa de actividad económica detrás de los trabajos que cuantificamos como respuesta final. Creemos que se necesitan nuevos estudios, como sugerimos a lo largo del texto, y algunos de los elementos enumerados en este artículo podrían enriquecer nuevas investigaciones.

\section{Bibliografía}

Akerlof, G. (1970). The Market for 'Lemons': quality uncertainty and the Market Mechanism. The Quarterly Journal of Economics, 84 (3), pp. 488-500.

Arrow, K. J. (1974). The Limits of Organization. Norton. 
Azeredo, D. (2016). Na proa dos negócios: a inserção feminina nas transações de crédito fluminense no início do século XIX (1800-1820). Dissertação de Mestrado. Universidade Federal Rural do Rio de Janeiro. Recuperado

de: https://tede.ufrrj.br/jspui/bitstream/jspui/1779/6/2016\%20\%20Daiane\% 20Estevam\%20Azeredo.pdf

Bacellar, C. de A. P. (1997). Os senhores da terra: família e sistema sucessório de engenho no oeste paulista, 1765-1855. CMU/UNICAMP.

Barth, F. (1967). Game theory and Pathan Society. Man, 2 (4), pp. 629-647.

Barth, F. (1974). Esferas económicas en Darfur. En R. Firth (Org.) Temas de antropologia económica (pp. 150-174). Fondo de Cultura Económica.

Barth, F. (1981). Process and form in social life. Routledge \& Keagan Paul.

Berg, J., Dickhaut, J. y McCabe, K. (1995). Trust, reciprocity, and social history. Games and economic behavior, 10 (1), pp. 122-142.

Bohannan, P. (1959). The Impact of Money on an African Subsistence Economy. The Journal of Economic History, 19 (04), pp. 491-503.

Bohorquez, J. (2017). La confianza como retórica, el estatus como práctica: comerciantes estadunidenses y relaciones de agencia en el Caribe español (1798-1822). América Latina en la Historia Económica 24 (3), pp. 7-40. https://doi.org/10.18232/alhe.850.

Burt, D. R. (2001). Bandwidth and echo: Trust, information, and gossip in social networks. Citeseer.

Carrara, A. (2020). O crédito no Brasil no período colonial: Uma revisão historiográfica. Varia Historia, 36 (70), pp. 15-51. https://doi.org/10.1590/0104-87752020000100002.

Cerutti, S. (1998). A construção das categorias sociais. En J. Boutier y D. Julia Passados recompostos (pp. 233-242). Editora da Universidade Federal do Rio de Janeiro.

Dasgupta, P. (1988) Trust as a Commodity. En D. Gambetta (Ed.).Trust: Making and Breaking Cooperative Relations (pp. 49-72). Blackwell.

Espírito Santo, C. (2011). A alma é o segredo do negócio... e do crédito: Religião, costume, poder e economia no Império Português - Lisboa, Vila Rica e São Luís do Maranhão, Século XVIII. Actas del XXVI 
Simpósio Nacional de História. Recuperado de: http://www.snh2011.anpuh.org/resources/anais/14/1300934222_ARQ UIVO_TextoANPUH2011.pdf.

Feyerabend, P. (2011). Contra o método. Edição 2. Editora Unesp.

Florentino, M. (1997). Em costas negras: uma história do tráfico de escravos entre a África e o Rio de Janeiro - séculos XVIII e XIX. Companhia das Letras.

Fragoso, J. y Florentino, M. (2001). O Arcaísmo como Projeto: mercado atlântico, sociedade agrária em uma economia colonial tardia. Civilização Brasileira.

Gil, T. (2015). Redes e camadas de relacionamentos na economia: metodologias para o estudo da confiança mercantil na América Portuguesa do Antigo Regime. Revista de Indias, LXXV (264), pp. 421-456. https://doi.org/10.3989/revindias.2015.014

Gil, T. (2018). Práticas creditícias e o cofre dos órfãos na vila de Curitiba (1780-1810). En C. G. Guimarães e L. F. Saraiva (Orgs.). Crédito e descrédito: relações sociais de empréstimos na América - Séculos $X V I I I$ ao XX (pp. 56-87). Eduff.

Gil, T. (2020). Coisas do caminho: crédito, confiança e informação na economia do comércio de gado entre Viamão e Sorocaba (17801810). Editora da UnB.

Gil, T. e Pesavento, F. (2014). Por ser público e notório': notas sobre informação na economia da América Lusa (séculos XVIII e XIX). Locus: Revista de História, 20 (2). https://periodicos.ufjf.br/index.php/locus/article/view/20772.

Glaeser, E.L; Laibson, D.; Scheinkman, J. y Soutter; C. (1999). What is Social Capital? The Determinants of Trust and Trustworthiness. Working Paper, 7216. https://doi.org/10.3386/w7216.

Gribaudi, M. y Blum, A. (1990). Des catégories aux liens individuels: l'analyse statistique de l'espace social. Annales, 45 (06), pp. 13651402 .

Guinnane, T. W. (2001). Cooperatives as Information Machines: German Rural Credit Cooperatives, 1883-1914. The Journal of Economic History, 61 (2), pp. 366-389. 
Guinnane, T. W. (2005). Trust: a concept too many. Jahrbuch für Wirtschaftsgeschichte/Economic History Yearbook, 46 (1), pp. 77-92. https://doi.org/10.1524/jbwg.2005.46.1.77.

Imízcoz Beunza, J. M. (2004). Actores, redes, procesos: reflexiones para una historia más global. Revista da Facultade de Letras- História, 5, pp. $115-140$

Johnson, N. D. e Mislin, A. A. (2011). Trust Games: A Meta-Analysis. Journal of Economic Psychology 32 (5), pp. 865-889. https://doi.org/10.1016/j.joep.2011.05.007.

Korczynski, M. (2000). The Political Economy of Trust. Journal of Management Studies, 37 (1), pp. 1-21.

Levi, G. (2000). A Herança Imaterial: trajetória de um exorcista no Piemonte do século XVII. Civilização Brasileira.

Lima, F. C. G. de C. (2012). A escassez de numerário e a adoção do açúcar como moeda no Brasil colonial. Revista Econômica, 14 (1), pp. 63-71.

Malaquias, C. de O. (2014). Remediados senhores: pequenos escravistas na Freguesia de São José do Rio das Mortes (c. 1790-c. 1844). Doutorado, Universidade Federal de Minas Gerais. Recuperado de: https://repositorio.ufmg.br/bitstream/1843/BUOS9UQRMA/1/tese_carlos.malaquias_versao.final_.pdf

Manski, C. F. (2000). Economic analysis of social interactions. Journal of economic perspectives 14 (3), pp. 115-136.

Mathias, C. L. K. (2012). As múltiplas faces da escravidão. Mauad Editora Ltda.

Moraes, L. S. de (2018). As viúvas da Vila de Curitiba e suas estratégias econômicas (1770-1800). Dissertação (Mestrado em História)Universidade de Brasília, Brasília. Recuperado de: https://repositorio.unb.br/bitstream/10482/35119/1/2018_LanaSatode Moraes.pdf

North, D. C. (1994). Estructura y cambio en la historia económica. Alianza Editorial.

Novaes Marques, T. (2014). Eram os senhores de engenho caloteiros? Reflexões sobre o crédito e os direitos de propriedade no mundo luso. História Econômcia \& História de Empresas, XVII, pp. 147-176. https://doi.org/10.29182/hehe.v17i1.259. 
Ogilvie, S. (2005). The Use and Abuse of Trust: Social Capital and its Deployment by Early Modern Guilds. Jahrbuch für Wirtschaftsgeschichte/ Economic History Yearbook, 46 (1). https://doi.org/10.1524/jbwg.2005.46.1.15.

Pereira, M. R. de M. e Navarro Borges, J. (2010). Tudo consiste em dívidas, em créditos e em contas: relações de crédito no Brasil colônia; Curitiba na primeira metade do século XVIII. Revista de História, (162), pp. 105-129.

Pesavento, F. (2013). Um pouco antes da Corte: a economia do Rio de Janeiro na segunda metade do setecentos. Paco Editorial.

Pesavento, F. (2018). Até que a confiança nos separe: as redes transimperiais e o mercado de crédito do Rio de Janeiro durante a segunda metade do século XVIII. En C. G. Guimarães e L. F. Saraiva (Orgs.). Crédito e descrédito: relações sociais de empréstimos na América - Séculos XVIII ao XX (pp. 18-55). Eduff.

Pinheiro, A. do C. e S. (2018). Hipoteca de Escravos no Sistema de Crédito Colonial Brasileiro - Vila de Curitiba (1773 - 1812). Monografia de Graduação, Universidade de Brasília. Recuperado de: https://bdm.unb.br/bitstream/10483/22684/1/2018_AmandaDoCoutoSi IvaPinheiro_tcc.pdf

Puntoni, P. (2017). O Estado do Brasil: poder e política na Bahia colonial1548-1700. Alameda Casa Editorial.

Ribeiro, A. V. (2009). A cidade de Salvador: estrutura econômica, comércio de escravos, grupo mercantil (c. 1750-c. 1800). Doutorado, Universidade Federal do Rio de Janeiro. Recuperado de: https://www.livrosgratis.com.br/ler-livro-online-77435/a-cidade-desalvador--estrutura-economica-comercio-de-escravos-grupomercantil-c1750--c1800

Rosenthal, J-L., Hoffman, G. e Postel-Vinay, G. (2000). Priceless Markets: the Political Economy of Credit in Paris, 1660-1870. The University Chicago Press.

Sampaio, A. C. J. de. (2003). Na encruzilhada do Império: hierarquias sociais e conjunturas econômicas no Rio de Janeiro (c.1650 -c.1750). Arquivo Nacional.

Santos, R. F. (2003). Trânsito material e práticas creditícias na América Portuguesa - Comarca do Rio das Velhas, Minas Gerais, século XVIII. 
Anais da $V$ Jornada Setecentista. Curitiba. Recuperado de: http://www.humanas.ufpr.br/portal/cedope/files/2011/12/Tr\%C3\%A2ns ito-material-e-pr\%C3\%A1ticas-credit\%C3\%ADcias-na-

Am\%C3\%A9rica-Portuguesa-Comarca-do-Rio-das-Velhas-MinasGerais-sXVIII-Raphael-Freitas-Santos.pdf

Santos, R. F. (2005). "Devo que pagarei": sociedade, mercado e práticas creditícias na comarca do Rio das Velhas-1713-1773. Mestrado, Universidade Federal de Minas Gerais. Recuperado de: https://www.academia.edu/19347976/_Devo_que_pagarei_sociedade mercado_e_pr\%C3\%A1ticas_credit\%C3\%ADcias_na_comarca_do_ Rio_das_Velhas_1713_1773

Santos, R. F. (2013). Minas com Bahia: mercados e negócios em um circuito mercantil setecentista. Doutorado, Universidade Federal Fluminense. Recuperado de: https://www.historia.uff.br/stricto/td/1445.pdf

Souza, E. S. de (2019). O mercado de crédito na Corte joanina: experiências das relações sociais de empréstimos (c. 1808-1821). Eduff.

Stiglitz, J. E. (1990). Peer Monitoring and Credit Markets. The World Bank Economic Review, 4 (3), pp. 351-66.

Trivellato, F. (2009). The familiarity of strangers: the Sephardic diaspora, Livorno, and cross-cultural trade in the early modern period. Yale University Press.

Trujillo, O. (2020). Francisco Álvarez Campana. Negocios, inversiones y sociedad en el Buenos Aires colonial (1750-1773). Prohistoria.

Valencia, C. (2018). Pequeños acreedores de grandes deudores: clientes y acreedores de las casas bancarias cariocas en la quiebra de 1864 . En C. G. Guimarães e L. F. Saraiva (Orgs.). Crédito e descrédito: relações sociais de empréstimos na América - Séculos XVIII ao XX. (pp. 86-121). Eduff.

Wasserman, M. (2018). Las obligaciones fundamentales: crédito y consolidación económica durante el surgimiento de Buenos Aires. Prometeo Libros.

Williamson, O. E. (1993). Calculativeness, trust, and economic organization. The journal of law and economics, 36 (1), pp. 453-486 
Yunus, M. (2004). Grameen Bank, Microcredit and Millennium Development Goals. Economic and Political Weekly, 39 (36), pp. 4.077-4.080.

Zucker, L.G. (1977). The role of institutionalization in cultural persistence. American Sociological Review, 42 (5), pp. 726-743. 